\section{Sharp increase in influenza A infections in Limpopo: A call for increased influenza vaccinations}

To the Editor: Influenza is often characterised by yearly seasonal epidemics and sporadic outbreaks, which are typically caused by influenza virus types A, B and $\mathrm{C}^{[1]}$ Influenza $\mathrm{A}$ and $\mathrm{B}$ are further classified into antigenically diverse subtypes, including influenza A (H1N1)pdm09, influenza A H3, influenza B Yamagata and influenza B Victoria. These viruses cause acute respiratory disease and are transmitted through aerosolised droplets and by direct contact with infected individuals or contaminated surfaces. Influenza A and B are responsible for the majority of seasonal influenza cases, and influenza A typically causes more morbidity and mortality. Those at highest risk of severe illness and death include the very young, the elderly and the immunocompromised. ${ }^{[2]}$

South Africa's influenza season typically occurs during the winter months ( May September) ${ }^{[3]}$ The 2021 influenza season coincided with the country's third COVID19 epidemic wave, raising the possibility of intersecting incidences of COVID-19 and influenza. Influenza and COVID-19 infections cause similar symptoms, yet have vastly different treatment and management approaches. As such, it is important to diagnose infections correctly when presented with overlapping symptoms. To do so, multiplex real-time polymerase chain reaction (PCR) assays that simultaneously detect multiple respiratory pathogens of interest can be used.

The Allplex SARS-CoV-2/FluA/FluB/ RSV assay (Seegene, South Korea) is a South African Health Products Regulatory Authority-approved real-time multiplex PCR assay that concurrently detects SARSCoV-2, influenza A, influenza $B$ and respiratory syncytial virus. Since June 2021, Ndlovu Laboratories in rural Limpopo Province has employed this assay to test all COVID-19 samples received at our site. As of 17 November 2021, we conducted a combined total of 7764 SARS-CoV-2/FluA/ FluB/RSV tests. During the past 2 months (October and November), we observed a sharp increase in the number of influenza A-positive samples. In October we recorded 60 positive samples compared with 8 in September, marking a 7.5-fold monthly increase. As of 17 November there had been 90 influenza A-positive test outcomes, signalling a potential outbreak (Fig. 1A). This increase is unexpected, given that the

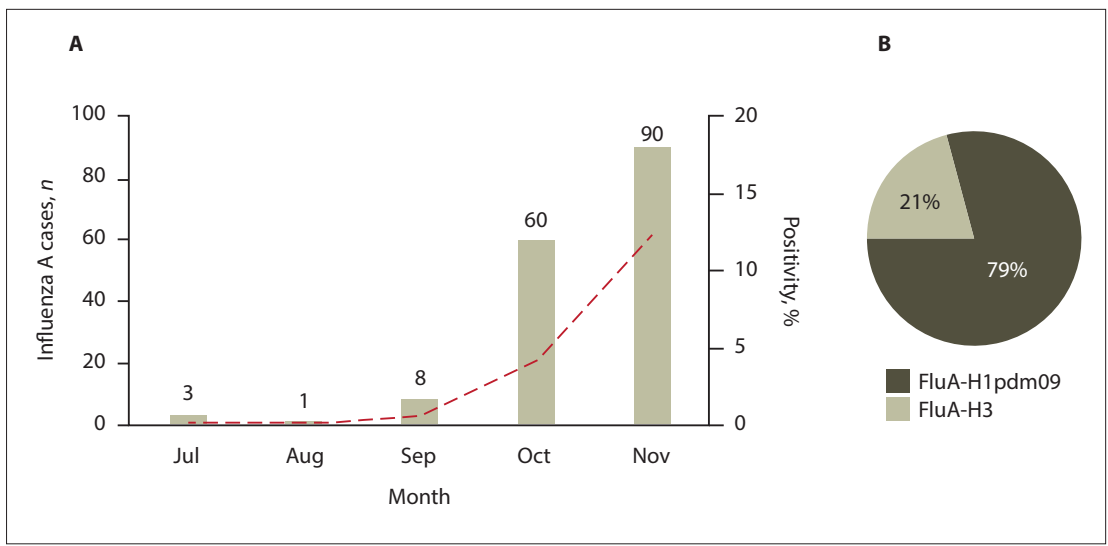

Fig. 1. Influenza A detection at Ndlovu Laboratories. (A) Influenza A cases detected from 1 July to 17 November 2021 (left y-axis). The dashed red line represents influenza A monthly percentage positivity (right y-axis). (B) Influenza A subtyping results: 48 randomly selected test positives from October and November cases were subtyped by real-time multiplex polymerase chain reaction.

influenza season is over by this time of the year. Of note, the increase in influenza $\mathrm{A}$ cases is not due to increased testing, as our number of tests declined monthly from June owing to decreased COVID-19 infections. Furthermore, there was a sharp increase in percentage positivity in our monthly testing, supporting increasing influenza $\mathrm{A}$ incidence (Fig. 1A, red dashed line).

We verified a random subset $(n=48)$ of October and November test positives by FluA subtyping. Our subtyping results indicate that the majority of the cases were of subtype H1N1pdm09 (Fig. 1B: H1N1pdm09 - $n=38 / 48$; H3 - $n=10 / 48$ ). The majority of the confirmed samples were from Limpopo $(n=44 / 48)$, with the remainder being from North West Province. Symptoms typical of influenza were reported for 42 of the 48 confirmed cases, with cough $(n=24 / 42)$, fever $(n=22 / 42)$, headache $(n=14 / 42)$, body aches $(n=13 / 42)$, sore throat $(n=12 / 42)$ and runny nose $(n=7 / 42)$ being most commonly reported. Taken together, these results reflect a substantial rise in influenza A cases despite the influenza season being historically over. These findings have been reported to the National Institute for Communicable Diseases (NICD), which is currently performing follow-up investigations and more in-depth surveillance. ${ }^{[4]}$

The 2021 influenza season commenced after more than a year of nationwide lockdown measures comprising social gathering restrictions, strict masking regulations and implementation of other non-pharmaceutical interventions (NPIs) in response to the COVID-19 pandemic. These measures were extremely important in reducing exposure to and limiting the spread of SARS-CoV-2. We speculate that these measures also limited exposure to influenza viruses, indirectly resulting in decreased immunity in the population. As COVID-19 restrictions are being lifted and adherence to NPIs decreases, respiratory pathogens may begin to recirculate. Our data are consistent with an immunity gap in the population resulting in increased influenza infections as COVID-19 restrictions and use of NPIs reduce. ${ }^{[4]}$ To mitigate such a scenario and prevent influenza outbreaks, we strongly recommend increased vaccinations against influenza in addition to ongoing COVID19 vaccination efforts. This is especially crucial for high-risk individuals, including children, the elderly, pregnant women, the immunocompromised, individuals with comorbidities, healthcare workers and employees who work in close proximity to each other, as well as for the general public. Special efforts must be made to ensure that national surveillance for respiratory pathogens of concern are not neglected during the COVID-19 pandemic, for example by increasing public-private partnerships between the NICD and laboratories across the country, which could act as additional sentinel sites. Finally, the NICD should be duly alerted to instances of increased cases of respiratory infections to detect infection waves early and contain them more easily.

\section{N Umunnakwe}

Ndlovu Research Centre, Ndlovu

Laboratories, Elandsdoorn, Limpopo

Province, South Africa

cumunnakwe@ndlovu.com

\section{Z N Makatini}

Department of Virology, Faculty of Health Sciences, University of the Witwatersrand, Johannesburg, South Africa 


\section{R Maphoto}

Department of Virology, Sefako Makgatho Health Sciences University, Pretoria, South Africa

\section{H A Tempelman}

Ndlovu Research Centre, Ndlovu Laboratories, Elandsdoorn, Limpopo Province, South Africa

\footnotetext{
1. World Health Organization. Influenza (seasonal). https://www.who.int/news-room/fact-sheets/detail/ influenza-(seasonal) (accessed 19 November 2021).
}

2. Cohen C, Kleynhans J, Moyes J, et al. Asymptomatic transmission and high community burden of seasonal influenza in an urban and a rural community in South Africa, $2017-18$ (PHIRST): A population cohort study. Lancet Glob Health 2021;9(6):E863-E874. https://doi.org/10.1016/S2214109X(21)00141-8

3. Bishop K, McMorrow M, Meiring S, et al. An evaluation of an influenza vaccination campaign targeting pregnant women in 27 clinics in two provinces of South Africa, 2015 - 2018. BMC Health Serv Res 2021;21:941. https://doi.org/10.1186/s12913-021-06962-8

4. National Institute for Communicable Diseases. Alert: Increase in influenza cases in South Africa. 17 November 2021. https://www.nicd.ac.za/alert-increase-in-influenza-cases-in-south-africa/ (accessed 18 November 2021).

S Afr Med J 2022;112(1):3-4. https://doi.org/10.7196/SAMJ.2022.v112i1.16284 